\title{
Safe-Nav: learning to prevent PointGoal navigation failure in unknown environments
}

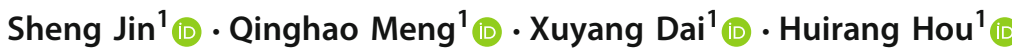

Received: 18 September 2021 / Accepted: 1 January 2022 / Published online: 21 January 2022

(c) The Author(s) 2022

\begin{abstract}
Training robots to safely navigate (Safe-Nav) in uncertain complex environments using the RGB-D sensor is quite challenging as it involves the performance of different tasks such as obstacle avoidance, optimal path planning, and control. Traditional navigation approaches cannot generate suitable paths which guarantee enough visible features. Recent learning-based methods are still not mature enough due to their proneness to collisions and prohibitive computational cost. This paper focuses on generating safe trajectories to the desired goal while avoiding collisions and tracking failure in unknown complex environments. We present Safe-Nav, a hierarchical framework composed of the visual simultaneous localization and mapping (SLAM) module, the global planner module and the local planner module. The visual SLAM module generates the navigation map and the robot pose. The global planner module plans a local waypoint on the real-time navigation map. In the local planner module, a deep-reinforcement-learning-based (DRL-based) policy is presented for taking safe actions towards local waypoints. Our DRL-based policy can learn different navigation skills (e.g., avoiding collisions and avoiding tracking failure) through specialized modes without any supervisory signals when the PointGoal-navigation-specied reward is provided. We have demonstrated the performance of our proposed Safe-Nav in the Habitat simulation environment. Our approach outperforms the recent learning-based method and conventional navigation approach with relative improvements of over 205\% ( 0.55 vs. 0.18 ) and $139 \%$ ( 0.55 vs. 0.23$)$ in the success rate, respectively.
\end{abstract}

Keywords Visual navigation · Deep reinforcement learning · Tracking failure

\section{Introduction}

In the research of robot autonomous navigation, visual navigation has gradually become a primary and promising research direction with the rapid development of robotic vision [1,2]. Visual navigation tasks can be divided into many forms. For example, PointGoal navigation defines a task where an intelligent robot equipped with a visual/RGB$\mathrm{D}$ sensor should navigate to the target location, provided by the relative position to the robot without prior maps [3,4]. In the PointGoal navigation task, the robot must learn a good environment representation and be robust to state-estimation errors in unknown environments. Therefore, PointGoal navigation can also reflect the automation level of the robotic system.

Xuyang Dai

dxy1993@tju.edu.cn

1 School of Electrical and Information Engineering, Tianjin University, Tianjin 300072, China
The traditional navigation pipeline uses a modular and hierarchical fashion to decompose the navigation task into a series of subtasks, such as localization, mapping and planning [5]. The robot relies on the visual/RGB-D sensor to map and localize within the environment and then plan a suitable route from the starting position to the desired goal [6-8]. Localization and mapping using visual simultaneous localization and mapping (SLAM) methods are fundamental for correct visual navigation. Recently, visual SLAM systems have been widely studied in the literature [9-11]. However, visual SLAM systems still suffer from robustness issues. For example, feature-based visual SLAM systems often suffer from tracking failure in low-texture scenes due to the insufficient visible features, which can result in inaccurate pose estimation. Path planning is also a key component for visual navigation, which is often composed of global planners [12,13] and local planners [14,15]. The global planners obtain the optimal path from the starting location to the target location based on the environmental map and robot pose. Dijkstra's algorithm and $\mathrm{A}^{*}$ algorithm are two 
widely used global planner algorithms. The former searches the map in a full range to obtain the optimal path, but a large amount of calculation is required, and the latter adds a heuristic term to accelerate the searching process of the algorithm [16]. With the increasing complexity and uncertainty of unknown environments, the efficiency of global path planning decreases significantly. Local planner computes the path through the local information, which has real-time planning ability. Dynamic Window Algorithm (DWA) [14] is a commonly used local planner algorithm, which only considers the environmental information within a speed window. From the sample range of speed, the trajectory with the highest score is selected as the output. Timed Elastic Band (TEB) [17] is also a widely used local path planning algorithm, which optimizes the constraints in the framework of General Graph Optimization (g2o) [15]. However, these methods require tuning numerous parameters manually. The second disadvantage of local planner algorithms is that they easily lead to tracking failure when combined with visual SLAM. The localization quality of visual SLAM is affected by the number of extracted feature points. Tracking failure easily occurs when there are not enough feature points observed in the current image, resulting in inaccurate pose estimation.

Currently, deep reinforcement learning (DRL) has shown tremendous potential in solving complex decision-making problems. DRL has spurred a lot of significant breakthroughs in many applications, such as manipulator controlling [18], autonomous driving [19,20], and games [21,22]. Considering the strengths of DRL, researchers attempt to apply it to tackle the PointGoal navigation problem [4,23-26]. Compared with the previous traditional methods, the DRL-based methods avoid extensive hand-engineering but only need to learn the complete navigation system directly from the data. Potentially, these methods can make the best use of the regularity in the data to achieve better performance than hand-crafted systems.

The methods in [23,27-30] use data-driven techniques to learn navigation strategies that can be deployed in previously unknown environments without the need of building an explicit spatial representation of the environment. However, these approaches are inherently sample-inefficient because the information provided by mature mapping, localization, and path-planning methods are ignored. Specially, Wijmans et al. [23] have trained their model to achieve good performance by using 2.5 billion frames of experience, which requires more than 6 months of GPU time! Although Ye et al. [27] propose that the sample efficiency can be improved using multiple auxiliary tasks, their training process still needs 40 M frames and 1 GPU-week. Meanwhile, some researchers propose to decouple the perception and policy to speed up the DRL training process [31-33]. However, the perceptual part also requires a lot of training and needs to be fine-tuned when facing a new scene.
Moreover, it is found that most learning-based models $[23,27,28,31-34]$ can use the simulator's flaws to slide along the obstacles instead of avoiding them $[29,35]$. Such policies may lead to catastrophic failures in the real world because collisions may damage the robot. Kadian et al. [29] find this flaw for the first time and modify the simulator to disable sliding along the obstacles. However, they only limit the number of collisions rather than learn how to avoid them. Sax et al. [30] and Bigazzi et al. [35] penalize obstacle collision with a large negative constant in the training process. However, Morad et al. [36] find that an effective collision-free policy cannot be learned when the negative constant is enforced in the training process due to reward sparsity and catastrophic forgetting [37-39]. To mitigate this elevated reward sparsity, they propose to use an automatic curriculum learning technique to improve the data sampling.

In summary, approaches to solve PointGoal navigation can broadly be classified into two categories: (1) traditional navigation methods that decompose navigation into localization, mapping and planning [12-15] or (2) learning neural policies using DRL [4,23-29,35]. Traditional navigation methods have the weakness of intensive computational demand and involve numerous parameters that need to be tuned manually. Moreover, these methods cannot constrain a path such that the robot can see enough features. For the learning-based methods, simultaneously implementing motion planning, scene layouts and obstacle avoidance are extremely challenging and prohibitively expensive.

Based on these problems, we propose a PointGoal navigation framework to train and integrate safe DRL-based policy into the traditional navigation pipeline. In our method, the PointGoal navigation framework comprises a visual SLAM module, a global planner module and a DRL-based local planner module. The visual SLAM module produces the robot pose and two-dimensional (2D) navigation map from raw sensory inputs. To achieve long-range navigation, the global planner module is used as a local waypoint generator that connects the visual SLAM module and the DRL-based local planner module. The global planner module consumes the 2D navigation map and the robot pose from the visual SLAM module, and uses a conventional global planning algorithm to generate local waypoints for the DRL-based local planner module. The DRL-based local planner module can directly map both the non-spatial input (i.e., the relative distance and relative angle) and spatial input (i.e., RGB image, depth image and feature points image) to safe actions towards local waypoints while avoiding collisions and tracking failure. Three specialized movement modes are included in the local planner module to ensure safety, each corresponding to a specific navigation skill (e.g., obstacles avoidance, tracking failure avoidance).

In summary, the main contributions of this paper are as follows: 
1. We introduce Safe-Nav, a hierarchical navigation framework to train and integrate the DRL-based method into the traditional robot navigation pipeline.

2. We combine three specialist movement modes in the local planner module to actively plan safe paths of a robot to a goal while avoiding collisions and tracking failure.

3. Extensive simulation results on complex and unseen scenarios, and systematic research on the safety and ablation demonstrate the effectiveness of our proposed framework.

\section{Methods}

\section{Overview}

Figure 1 presents a flowchart of our proposed framework Safe-Nav. The autonomous navigation system consists of a visual SLAM module (Nav-SLAM), a global planner module and a DRL-based local planner module. After sampling the start position and goal position, a PointGoal navigation task is started. Our visual SLAM module predicts the 2D navigation map of the environment and the robot poses from incoming RGB observations and depth observations in real time. The global planner module uses the predicted navigation map and the robot pose to generate a feasible trajectory from the current position to the goal position and select the local waypoint along the path. Next, the DRL-based local planner module executes the navigation process of moving to the local waypoint. Upon reaching the goal, colliding with the environment or happening tracking failure, the PointGoal navigation task ends.

The visual SLAM module, the global planner module and the DRL-based local planner module are closely connected in the proposed navigation framework Safe-Nav. The relationship between these three modules can be further elaborated as follows:

- The visual SLAM module is the basis of the navigation framework Safe-Nav. On the one hand, it provides the 2D navigation map and robot pose for the global planner module. On the other hand, it generates the feature point maps for the DRL-based local planner module as one of the spatial observation inputs.

- The global planner module is responsible for planning a local waypoint on the $2 \mathrm{D}$ navigation map based on the current robot pose. Then, the relative distance and angle between the current robot pose and the local waypoint are computed as the non-spatial observation inputs for the DRL-based local planner module.

- The DRL-based local planner module can directly map both non-spatial inputs and spatial inputs from the RGBD camera and other modules to safe actions towards local waypoints while avoiding collisions and tracking failure.

From section "Visual SLAM module" to section "DRLbased local planner module", each module is described in detail. In section "Evaluation metrics", we give a detailed description of the evaluation metrics. Then, in section "Simulation setup", we illustrate the simulation platform for training and evaluation.

\section{Visual SLAM module}

The visual SLAM module processes the RGB-D data in sequence and produces the robot pose and navigation map at each key frame. Due to the excellent performance of the Oriented FAST and Rotated BRIEF-SLAM2 (ORB-
Fig. 1 The overview of the proposed framework Safe-Nav. Each module is represented by a grey box. The DRL-based local planner module learns to approach a desired local waypoint while avoiding collisions and tracking failure

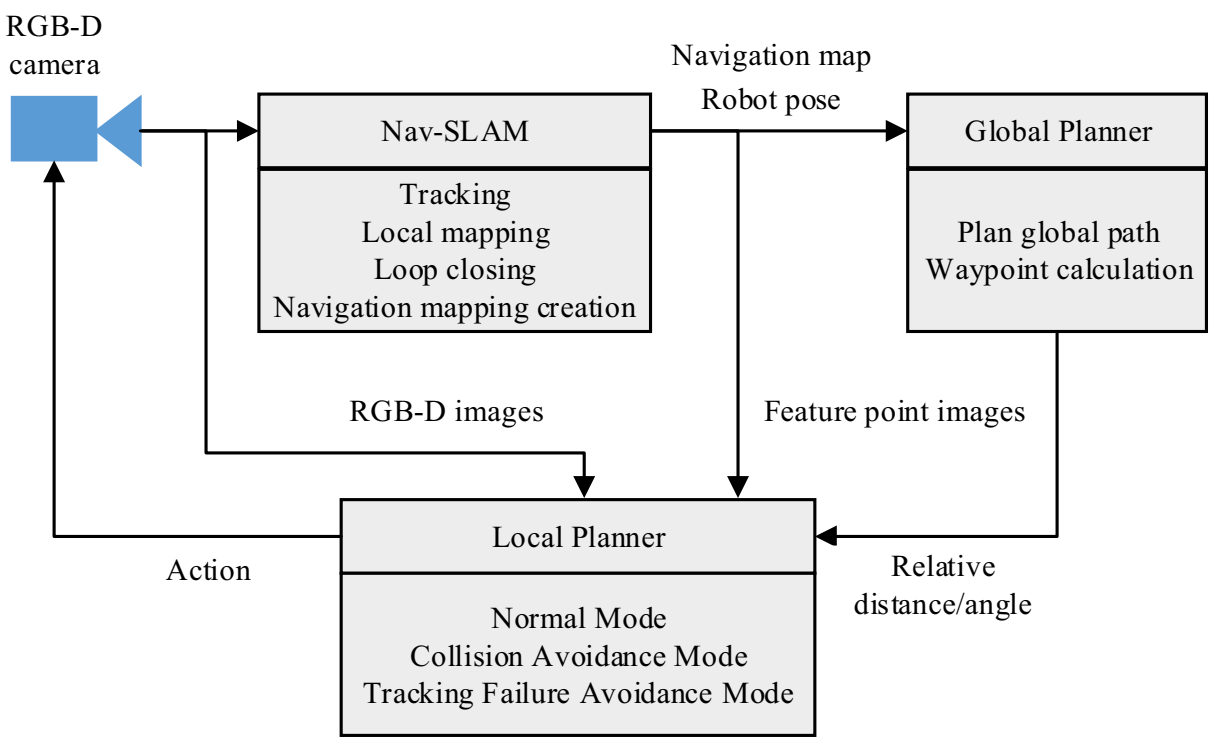


Fig. 2 The architecture of Nav-SLAM. The tracking thread, local mapping thread and loop closing thread are the same as ORB-SLAM2.

Compared with the original ORB-SLAM2 system, we extend the mapping method and reconstruct the environmental navigation map, which can be used in the PointGoal navigation tasks by introducing the navigation map creation thread

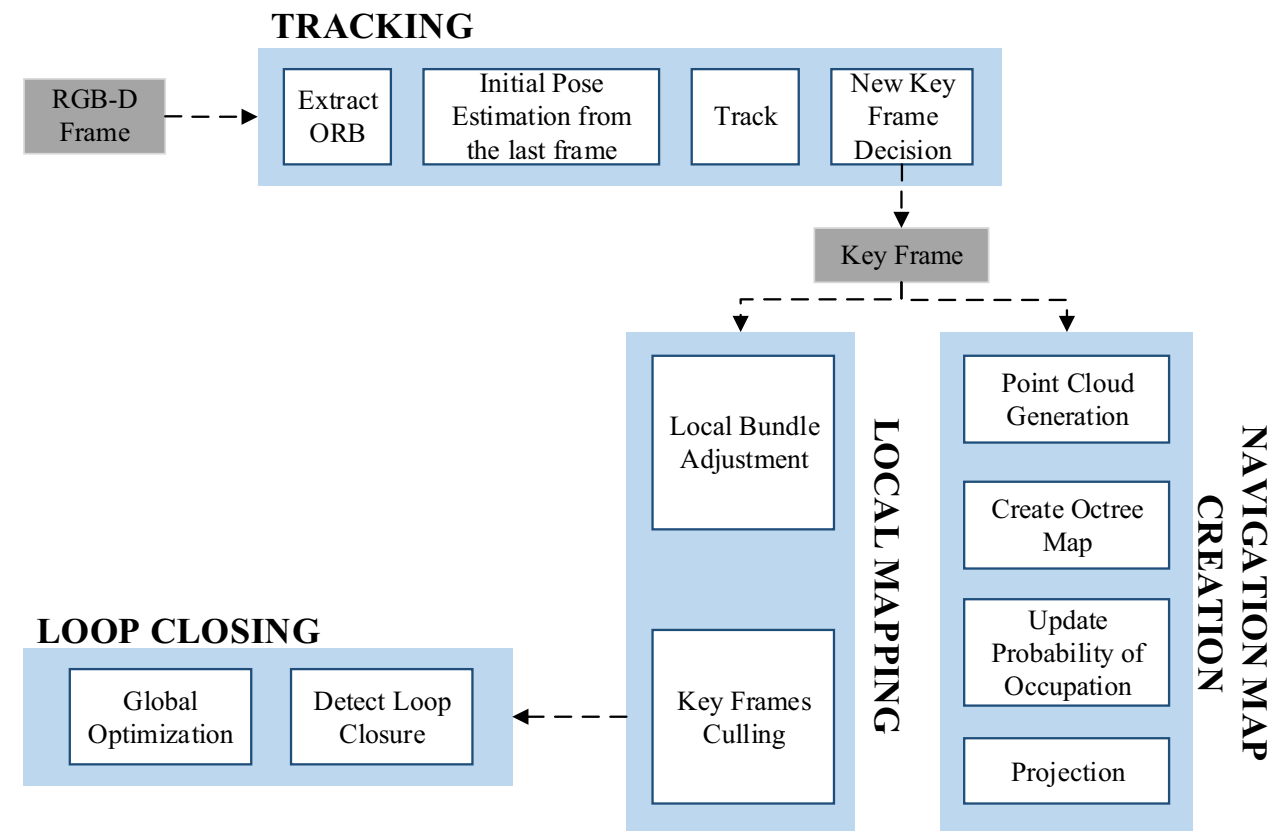

SLAM2) [40] in most application scenarios, it is adopted in our framework to provide a feature-based visual SLAM solution. However, the original ORB-SLAM2 method can only construct sparse point cloud maps, which only meets the needs of robot localization rather than robot navigation. Therefore, a complete visual SLAM system for the robot navigation task, named Nav-SLAM, is proposed based on ORB-SLAM2, enabling us to estimate the robot pose and produce the navigation map. The architecture of Nav-SLAM is shown in Fig. 2.

Four threads run in parallel in Nav-SLAM: tracking, local mapping, loop closing and navigation map creation. The navigation map creation thread gets new key frames and the key frames' poses from the tracking thread. The key frames' poses and the RGB-D images are used to generate the point cloud. Based on the camera projection model and the key frames' poses, the coordinate of the three-dimensional (3D) point corresponding to each pixel from the key frame in the world coordinate system can be obtained [41]. The spatial point $P_{c}=\left(x_{c}, y_{c}, z_{c}\right)$ in the camera coordinate system, which corresponds to the pixel coordinate $(u, v)$, can be expressed as

$\left\{\begin{array}{l}x_{c}=\frac{z_{c}}{f_{x}}\left(u-c_{x}\right) \\ y_{c}=\frac{z_{c}}{f_{y}}\left(v-c_{y}\right), \\ z_{c}=\frac{d}{s}\end{array}\right.$

where $c_{x}, c_{y}, f_{x}$ and $f_{y}$ are internal camera parameters. $d$ stands for the depth of the pixel (the distance from the pixel to the imaging plane). $s$ indicates the depth scale.
To obtain the coordinates of the pixels in the real world, it is necessary to use the pose of the key frame to transform $P_{c}$ into the coordinate in the world coordinate system $P_{w}$ :

$P_{w}=T_{i} \cdot P_{c}$

where $T_{i}$ is the pose of the $i$-th key frame. Performing the same operation on all pixels in each key frame can generate a 3D dense point cloud map of the environment.

Compared with the point cloud map, the octree map stores the map in an octree hierarchical structure [42], which saves more memory space than the point cloud map. Meanwhile, the octree map also has excellent properties such as adjustable resolution and updating nodes in probability. In the process of creating the point cloud map, we construct the octree map of the 3D environment in real time based on the octree map library. Then, we project the 3D octree map onto the $2 \mathrm{D}$ plane. Only those obstacles within a range of $[0.3,1.2]$ times the camera height $(1.25 \mathrm{~m})$ are projected to create the 2D navigation map, which avoids suspended objects or ground planes well.

\section{Global planner module}

This module aims to determine the global path from the current position to the target location along the local waypoints on the path. As there is no initial information about the environment, it is impossible to obtain the optimal global path or even ensure that the robot can successfully complete the PointGoal navigation task without collisions and tracking failure by simply following the global path. Therefore, to accomplish long-distance PointGoal navigation, it is neces- 
sary to find intermediate points from the global path as local waypoints for the local planner. Considering that $A^{*}[43]$ is a simple and efficient global path planning algorithm, we use $\mathrm{A}^{*}$ as the global planner to compute the global path. Given the global path and the current robot's localization, a local waypoint is selected along the path that lies at a distance of $d_{s}$ (which is set to be $0.6 \mathrm{~m}$ ) from the robot's current position.

\section{DRL-based local planner module}

\section{Problem formulation}

The task of the DRL-based local planner module is to navigate successfully from the current position to the local waypoint as fast as possible without collisions and tracking failure. The robot interacts with the environment by executing the translation velocity $(v)$ and rotation velocity $(\omega)$. We formulate this process as a partially observable Markov decision problem (POMDP), which can be defined as the 6-tuple ( $S, O, A, T, R, \gamma)$. Here, $S$ represents the robot's states, $A$ stands for the action space, $O$ is the observation space, $T$ indicates the transition probability from one state to another, $R$ means the reward and $\gamma$ expresses the discount factor. The robot cannot fully obtain the state $s_{t}$, so it has to rely on observations $o_{t} \in O$ (e.g., an RGB image). The state $s_{t} \in S$ changes based on the robot's actions $a_{t} \in A$ according to the transition probability $T\left(s_{t+1} \mid s_{t}, a_{t}\right)$, where $a_{t} \sim \pi\left(\cdot \mid o_{t}\right)$. After each state transition, the robot receives a reward $r_{t}$. The goal of DRL is to find the optimal policy $\pi^{*}$ that maximizes the expected reward as follows:

$\pi^{*}=\operatorname{argmax} E_{T \sim \pi}\left[\sum_{t=1}^{T} \gamma^{t-1} r_{t}\right]$,

where the discount factor $\gamma$ balances the trade-off between exploration and exploitation.

In this paper, we use an on-policy gradient algorithm: Proximal Policy Optimization (PPO) [44] to find the optimal policy $\pi^{*}$. Given a series of collected trajectories (commonly named as 'rollout') and a $\theta$-parameterized policy $\pi_{\theta}$, the policy $\pi_{\theta}$ can be updated using PPO algorithm as follows. Let $\hat{A}_{t}=R_{t}-\hat{V}_{t}$ be the advantage function, where $R_{t}=\sum_{i=t}^{T} \gamma^{i-t} r_{i}$ and $\hat{V}_{t}$ is the expected value of $R_{t}$. Let $r_{t}(\theta)=\pi_{\theta} / \pi_{\theta_{\text {old }}}$ be the ratio of the current policy and the old policy used to collect the rollout, which can be used to achieve stability. Here, $\pi_{\theta_{\text {old }}}$ is the policy before the update. To avoid large policy updates, the ratio $r_{t}(\theta)$ is constrained to the range of $[1-\varepsilon, 1+\varepsilon]$ via the clip term. Then, the policy parameters are updated by maximizing the object function:

$$
\begin{aligned}
& \eta^{C L I P}(\theta) \\
& =E_{t}\left[\min \left(r_{t}(\theta) \hat{A}_{t}, \operatorname{clip}\left(r_{t}(\theta), 1-\varepsilon, 1+\varepsilon\right) \hat{A}_{t}\right)\right],
\end{aligned}
$$

where the expectation $E_{t}[\ldots]$ indicates the empirical average over the rollout.

\section{Observation space}

There are five different observation types which can be described as follows. The first type and the second type are the RGB images (denoted by $o_{r g b}$ ) and the depth images (denoted by $o_{\text {depth }}$ ), respectively. The third type is the relative distance between the local waypoint and the robot's position, which can be denoted as $o_{d i s}$. Given the coordinate position of the robot on the navigation map $p_{\text {robot }}=$ $\left(x_{1}, y_{1}\right)$ and the coordinate position of the local waypoint $p_{\text {waypoint }}=\left(x_{2}, y_{2}\right), o_{\text {dis }}$ can be computed as follows:

$o_{d i s}=\sqrt{\left(x_{2}-x_{1}\right)^{2}+\left(y_{2}-y_{1}\right)^{2}}$.

The fourth type is the relative angle between the local waypoint and the robot's position, which can be denoted as $o_{\text {angle }}$. Given the deflection angle in the world coordinate system $A_{\theta}, o_{\text {angle }}$ can be computed as follows:

$o_{\text {angle }}=A_{\theta}-A_{\phi} \pm 2 \pi$, where $A_{\phi}=\frac{y_{2}-y_{1}}{x_{2}-x_{1}}$,

where $2 \pi$ constrain the relative angle $o_{\text {angle }}$ within $[-\pi, \pi]$. If $A_{\theta}-A_{\phi}$ is larger than $\pi, 2 \pi$ is subtracted. If $A_{\theta}-A_{\phi}$ is smaller than $-\pi, 2 \pi$ is added.

In addition, we use a representation of ORB feature points in the observation. In ORB-SLAM2, ORB features [45] are used for tracking. The performance of ORB-SLAM2 is greatly affected by the number of ORB feature points in the observed images, because the small number of feature points can lead to inaccurate pose estimation. In each step, we extract ORB feature points from the RGB image. Then, the binary feature point image $o_{f p m}$ is constructed, which serves as the fifth observation type. Each pixel on $o_{f p m}$ is 0 or 1, which, respectively, represents the presence or absence of the ORB feature point.

\section{Action space}

In this paper, we train our DRL-based local planner module on a discrete action space composed of four discrete actions 〈move forward, turn left, turn right, move backwards〉 in total, which apply different translational and angular velocities to the agents. As mentioned earlier, tracking in visual SLAM systems usually fails during the period of pure rotational motions [46,47]. Handling pure rotational motion problem for visual SLAM has always been very challenging. To avoid this problem, our method avoids the pure rotational motion by applying a small translational velocity when turning left or right. Given the limits for the translational 
velocity $\left(-v_{\max }, v_{\min }, v_{\max }\right)$ and angular velocity $(-\omega, \omega)$, four discrete actions can be defined as: $\left(v_{\max }, 0\right),\left(v_{\min },-\omega\right)$, $\left(v_{\min }, \omega\right)$ and $\left(-v_{\max }, 0\right)$. The execution time of each action is $0.5 \mathrm{~s}$.

\section{Movement modes}

Avoiding collisions and tracking failure is important for our local planner module but are much harder to train simultaneously. Here, we propose three specialized movement modes: the normal mode, the collision-avoidance mode and the tracking-failure-avoidance mode. One mode might specialize in approaching the local waypoints fast, another in avoiding collisions and a third in getting enough visible features. By combining these modes, the robot can reach the local waypoints faster and learn to avoid collisions and tracking failure better.

At each time step, the robot's observation acts as a switch that selects a specialized mode. We assume a case in which the robot is very close to the obstacles. In this case, the robot's task is to approach a local waypoint safely while avoiding collisions. We define this movement mode as the collision-avoidance mode. Tracking failure can arise from observing limited visual features in featureless areas. We define the tracking-failure-avoidance mode, in which the robot is required to move to an area that can guarantee enough visual features. When the robot is far away from the obstacles and in a feature-rich area, the robot should be able to reach the local waypoint as fast as possible and try to avoid entering those dangerous areas. We define this case as the normal mode. During navigation, the normal mode is used more frequently than the other two modes.

Next, we will further describe the network architecture of each movement mode in detail.

1. Collision-avoidance mode. In this mode, a depth image along with the relative distance and angle between the robot position and the local waypoint, can be used as the input. In this way, a better capability of perceiving the distance to the obstacles and the relative position of the local waypoint can be available. Thus, we propose a two-branch feature extraction network that splits the input into non-spatial inputs (relative distance and relative angle) and spatial input (depth image). The non-spatial branch network is a single fully connected (FC) layer. It encodes the relative distance and angle into a high dimensional feature ( yellow rectangle in the upper branch in Fig. 3), further processed in the selfattention module. The depth image is fed into the spatial branch network to extract deep features, which is a ResNet18 [48] network structure (green layers in the left lower branch in Fig. 3. ResNet18 is a lightweight architecture and can achieve robust performance to the vanishing/exploding gradient problem during training, which has already shown its strengths in the task of visual navigation [49]. The adaptive average pooling layer replaces the output of the second last average pooling layer. The output of this layer is flattened and reduced to a one-dimensional output (green rectangle in the lower branch in Fig. 3), which is further processed in the following self-attention module.

Then, we concatenate the outputs of both branches and process them together in the following PPO-based neural network architecture, which is shown on the right side of Fig. 3. As mentioned above, a PPO-based neural network architecture is used to train the DRL-based local planner. PPO is an actor-critic network architecture. For the actor and critic networks, we use the same head. The output action space is discrete and consists of three actions - move forward, turn left, turn right. Rectified linear unit (ReLU) activation follows after the first two FC layers. The output of the last FC layer is used to produce a softmax distribution over the action space. In the critic network, ReLU activation is applied on all the FC layers. The output of the last FC layer is used to produce an estimate of the value function.

2. Tracking-failure-avoidance mode. It is important to avoid tracking failure in this mode instead of reaching the local waypoint since the number of associated visual features can affect the robot's localization capabilities. Less feature points often correlate to inaccurate relative position estimation by the visual SLAM module. Therefore, we only have visual sensing capabilities in the tracking-failure-avoidance mode, such as RGB images, depth images and feature point images, and lack any position sensing capabilities such as the relative distance and relative angle. We concatenate the outputs of the RGB image branch, depth image branch and the feature point image branch into a new feature, as shown in Fig. 4. Different from the collision-avoidance mode, we add the 〈move backwards〉 action in the tracking-failureavoidance mode, which can provide back and forth motions to better avoid tracking failure.

3. Normal mode. In this mode, the robot is required to move to the local waypoint in the shortest possible time and try to stay away from those dangerous areas that may lead to collisions and tracking failure. Therefore, a broader range sensing capability is required in this mode. The RGB$\mathrm{D}$ images, the feature point images, the relative distance and the relative angle are all used as the input, which is shown in Fig. 5.

\section{Self-attention modules}

Recently, the visual attention mechanism has been widely used in computer vision, making the deep learning network find the correlation between features and highlighting important features to improve the feature extraction ability [50-52].

For non-spatial input, i.e., the relative distance and relative angle, a common self-attention method is adopted [53]. We first feed the input to a fully connected layer, which encodes 
Fig. 3 The network architecture of the collision-avoidance mode

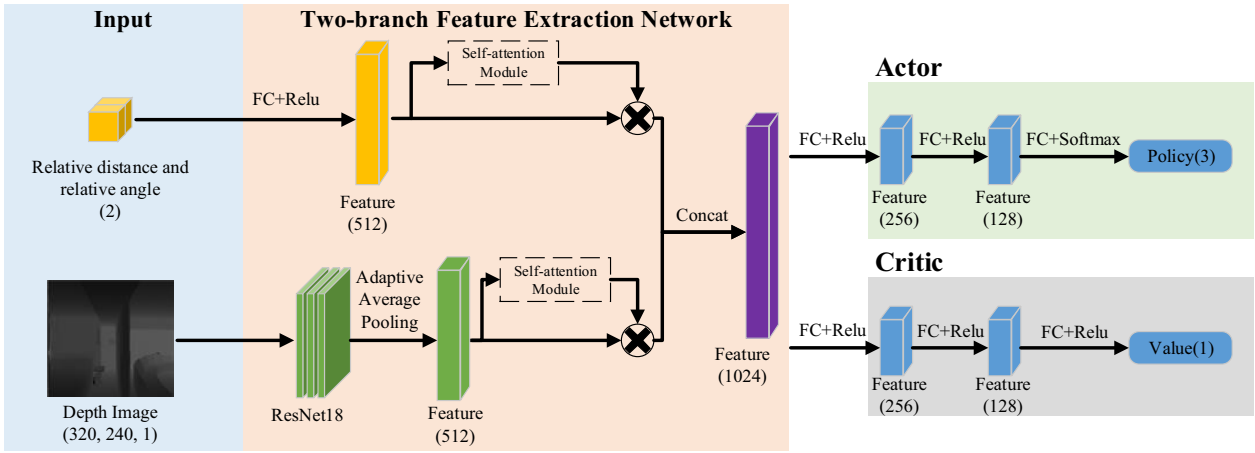

Fig. 4 The network architecture of the tracking-failure-avoidance mode
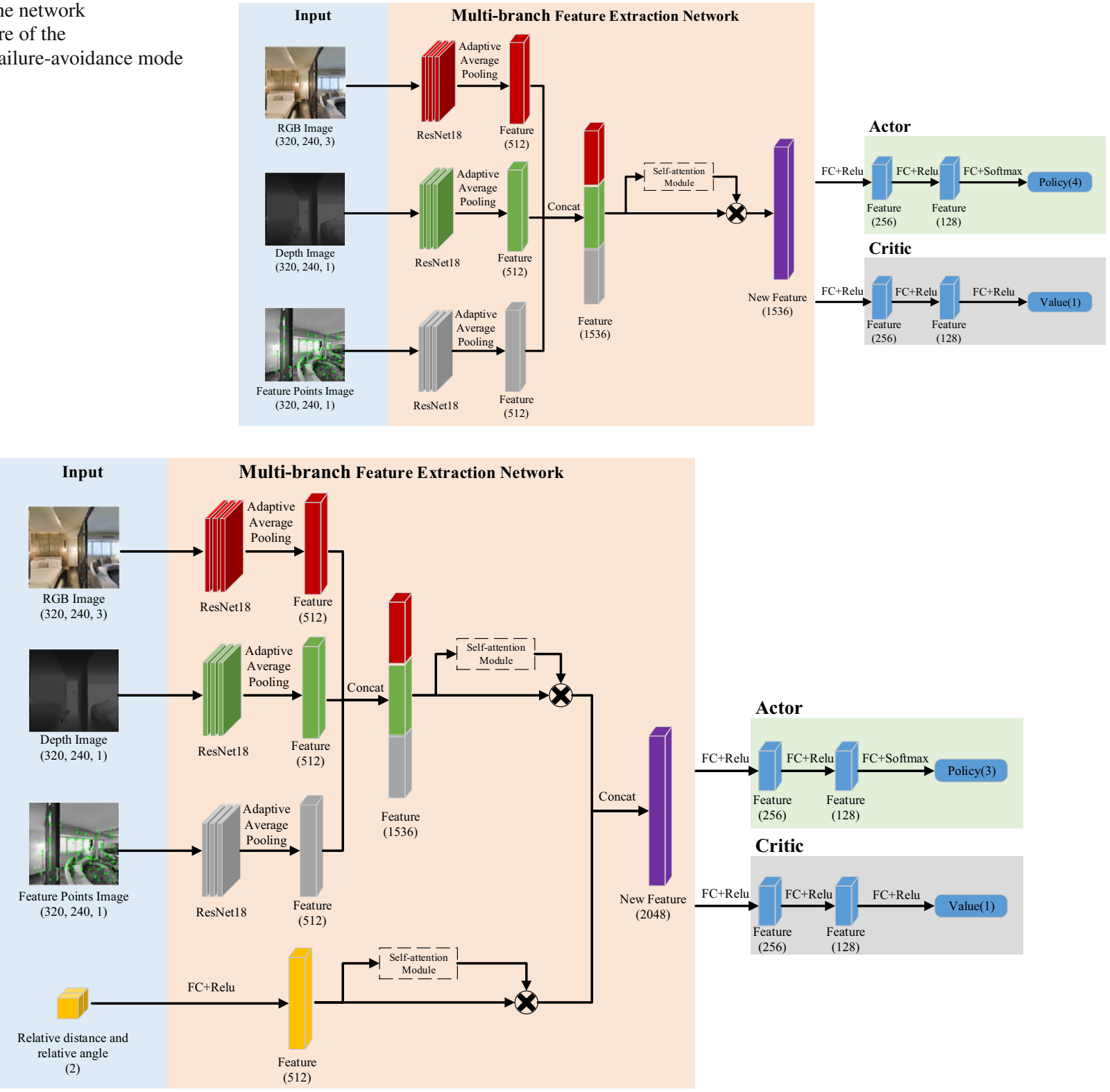

Fig. 5 The network architecture of the normal mode 


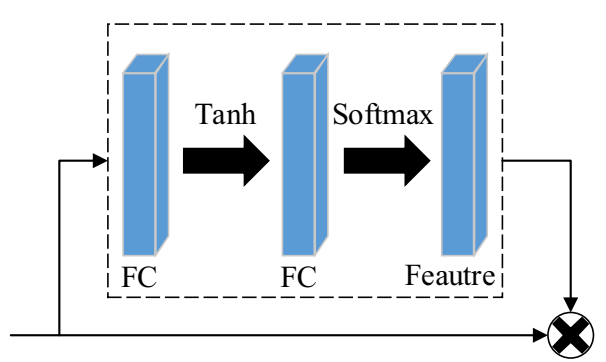

(a)

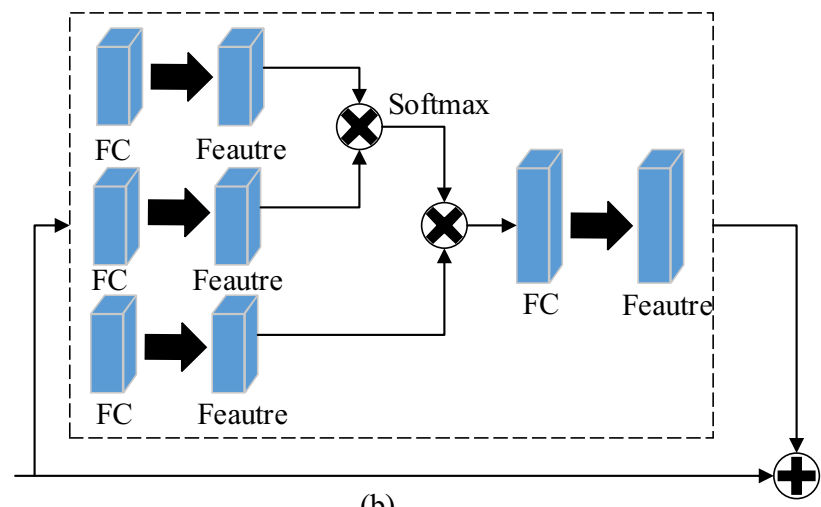

(b)

Fig. 6 The architecture of self-attention module. a Non-spatial input. b Spatial input

the relative distance and angle into a high dimensional feature space to process them in the following self-attention module. The self-attention module for non-spatial input is composed of two additional fully connected layers. We feed the obtained high dimensional features to the first fully connected layer followed by Tanh activation. The result is inserted into a second fully connected layer followed by a softmax activation. The produced feature weights and the original high dimensional features are combined through element-wise multiplication. The process is presented in Fig. 6a.

For the spatial input, such as RGB image, depth image and feature points image, we adopt a non-local style self-attention method, which has already been used in visual localization [54], video analysis [55] and image generation [56]. It can capture the long-range dependencies and global correlation of the image features, which is presented in Fig. 6b. Given a feature vector, its self-attention can be calculated using the softmax function. Then, the residual connection will be added to the linear embedding.

\section{Reward function}

1. In the normal mode, our reward function provides negative constants to discourage collisions and tracking failure, and positive rewards to encourage the robot to go to local waypoints as soon as possible. Therefore, our reward function considers five summands regarding the local waypoint $w p$, the relative distance $d s$, the relative angle $a g$, the obstacles $o$ and the tracking status of the visual SLAM module $t r$ :

$$
\begin{aligned}
R_{s a f e}^{t}= & R_{s a f e}^{t}(w p)+R_{s a f e}^{t}(d s)+R_{s a f e}^{t}(a g) \\
& +R_{s a f e}^{t}(o)+R_{s a f e}^{t}(t r)
\end{aligned}
$$

The reward $R_{\text {safe }}^{t}(w p)$ has a high positive reward $R_{w p}$ if the position of the robot $P_{r}^{t}$ reaches the local waypoint position $P_{w p}^{t}$ within radius $D_{w p}$ :

$R_{\text {safe }}^{t}(w p)= \begin{cases}R_{w p}, & \text { if }\left\|P_{r}^{t}-P_{w p}^{t}\right\|<D_{w p}, \\ 0, & \text { otherwise }\end{cases}$

The robot is rewarded for getting closer to the local waypoint $P_{w p}^{t}$, but it is punished for moving away from it. The $\operatorname{diff}(\cdot)$ function in Eq. (10) computes the difference between the relative distance at the previous time step and the current time step:

$R_{s a f e}^{t}(d s)=\omega_{1} \cdot \operatorname{diff}\left(P_{r}^{t}, P_{w p}^{t}\right)$

$\operatorname{diff}\left(P_{r}^{t}, P_{w p}^{t}\right)=\left\|P_{r}^{t-1}-P_{w p}^{t-1}\right\|-\left\|P_{r}^{t}-P_{w p}^{t}\right\|$.

Moreover, to reach the local waypoint as soon as possible, the robot should learn to adjust the relative angle and move towards the local waypoint. Therefore, given the relative angle at the previous time step $\alpha^{t-1}$ and the relative angle at the current time step $\alpha^{t}$, the robot is rewarded for reducing the relative angle and punished for increasing the relative angle, which can be expressed as

$R_{\text {safe }}^{t}(a g)=\omega_{2} \cdot\left(\alpha^{t-1}-\alpha^{t}\right)$

Of course, the robot is punished for colliding with obstacles. This enables the robot try to keep away from obstacles. If the robot collides with obstacles, it results in a high negative constant $R_{C O}$ :

$R_{\text {safe }}^{t}(o)= \begin{cases}-R_{C O}, & \text { if the robot collides with obstacles } \\ 0, & \text { otherwise }\end{cases}$

The robot is also punished for tracking failure. This allows the robot to avoid moving to areas where there are no features. If tracking failure occurs, the robot will get a high negative constant $R_{T F}$ :

$R_{\text {safe }}^{t}(t r)= \begin{cases}-R_{T F}, & \text { if tracking failure occurs } \\ 0, & \text { otherwise }\end{cases}$

2. In the collision-avoidance mode, our reward function provides positive rewards to encourage the robot to move away from obstacles and get closer to the local waypoints. 
We compute the minimum depth value $d_{\min }^{t}$ and the average depth value $d_{\text {avg }}^{t}$ from the depth image at each step. The robot is rewarded for increasing the minimum depth value, but it is punished for decreasing the minimum depth value in Eq. (14). To avoid the sparse reward when the robot turns right or turns left due to the small translational velocity, we reward the robot for increasing $d_{\text {avg }}^{t}$ [see Eq. (15)]. In addition, we limit the sum of $R_{o a}^{t}$ (mindepth) and $R_{o a}^{t}($ avgdepth) to $[-5,5]$ to avoid excessive changes of these rewards:

$R_{o a}^{t}($ mindepth $)=\left\{\begin{array}{c}\omega_{3} \cdot\left(d_{\text {min }}^{t-1}-d_{\text {min }}^{t}\right) \\ 0, \text { otherwise }\end{array}\right.$

$R_{\text {oa }}^{t}($ avgdepth $)=\left\{\begin{array}{c}\omega_{4} \cdot\left(d_{\text {avg }}^{t-1}-d_{\text {avg }}^{t}\right) \\ 0, \text { otherwise }\end{array}\right.$.

In addition, the robot is punished for colliding with obstacles. If the robot collides with obstacles, it results in a high negative constant $R_{C O}$ :

$R_{o a}^{t}(o)= \begin{cases}-R_{C O}, & \text { if the robot collides with obstacles } \\ 0, & \text { otherwise }\end{cases}$

Similarly as the normal mode, the robot is rewarded for getting closer to the local waypoint, but it is punished for moving away from it. It is important for the robot to pass through narrow areas:

$R_{o a}^{t}(d s)=\omega_{1} \cdot \operatorname{diff}\left(P_{r}^{t}, P_{w p}^{t}\right)$

$\operatorname{diff}\left(P_{r}^{t}, P_{w p}^{t}\right)=\left\|P_{r}^{t-1}-P_{w p}^{t-1}\right\|-\left\|P_{r}^{t}-P_{w p}^{t}\right\|$.

The robot is rewarded for reaching the local waypoint position $P_{w p}^{t}$ within the radius $D_{w p}$ without collisions:

$R_{o a}^{t}(w p)= \begin{cases}R_{w p}, & \text { if }\left\|P_{r}^{t}-P_{w p}^{t}\right\|<D_{w p} \\ 0, & \text { otherwise }\end{cases}$

The total reward of the collision-avoidance mode can be calculated as the sum of all sub-rewards:

$$
\begin{aligned}
R_{o a}^{t}= & R_{o a}^{t}(\text { mindepth })+R_{o a}^{t}(\text { avgdepth })+R_{o a}^{t}(o) \\
& +R_{o a}^{t}(d s)+R_{o a}^{t}(w p) .
\end{aligned}
$$

3. If the number of extracted ORB feature points is small during navigation in the normal mode, it will enter the tracking-failure-avoidance mode. There is no separate reward for successfully reaching the local waypoint as our aim is to avoid visual SLAM failure along the way rather than optimally reach the local waypoint. Therefore, our reward function in the tracking-failure-avoidance mode only considers three summands regarding the number of feature points $p o$, the tracking of the visual SLAM module $t r$ and the back motions back, as described as follows:

$R_{\text {track }}^{t}=R_{\text {track }}^{t}($ po $)+R_{\text {track }}^{t}($ tr $)+R_{\text {track }}^{t}($ back $)$.

Let $N_{\text {track }}^{t}$ be the number of feature points at the current time step. The robot is rewarded for obtaining more feature points, but it is punished for getting fewer feature points in Eq. (22). In addition, we limit $R_{\text {track }}^{t}$ (po) to [-6, 6] to avoid excessive changes of this reward:

$R_{\text {track }}^{t}($ po $)=\omega_{5} \cdot\left(N_{\text {track }}^{t-1}-N_{\text {track }}^{t}\right)$,

where $N_{\text {track }}^{t-1}$ and $N_{\text {track }}^{t}$ are the number of feature points visible at times $(t-1)$ and $t$, respectively.

Since the action space includes back motions in the tracking-failure-avoidance mode, it is dangerous for the robot to select continuous back motions. Since continuous back motions will easily lead to collisions. For that reason, the robot gets punished for selecting continuous back motions for some time steps $D_{b a c k}$ :

$R_{\text {track }}^{t}($ back $)=\left\{\begin{array}{l}-R_{\text {back }}, \text { if the robot moves } \\ \text { backward for duration Dack } \\ 0, \text { otherwise }\end{array}\right.$

Of course, the robot is punished for tracking failure and colliding with obstacles:

$R_{\text {track }}^{t}(t r)=\left\{\begin{array}{l}-R_{C O}, \text { if the robot collides with obstacles } \\ -R_{T F}, \text { if tracking failure occurs } \\ 0, \text { otherwise }\end{array}\right.$.

\section{Training}

Three modes were trained: the normal mode, the collisionavoidance mode and the tracking-failure-avoidance mode. The normal mode was first trained. For each PointGoal navigation task, the feasible start and goal positions were given. After obtaining the local waypoint position from the global planner module, a local planner process was started from the robot's current position to the local waypoint position. Each local planner episode was limited to $k$ time steps. Each local planner episode ended when the local waypoint was reached within the radius $D_{w p}$ or collision occurred or tracking failure occurred or a given time steps were reached. For each step, the obtained reward $r_{t}$ was stored into the buffer $R$. These values were used to calculate the value loss $L_{\text {value }}$ and the policy loss $L_{\text {policy }}$. Softmax was applied to obtain the policy values into probabilities of each action. Finally, the policy gradient and value gradient were calculated using 
the respective losses. The network parameters were updated accordingly. When either the goal was reached, or collision occurred, or tracking failure occurred, the PointGoal navigation task was terminated. Suppose $N$ PointGoal navigation tasks were given, the training algorithm can be described in Algorithm 1 shown below.

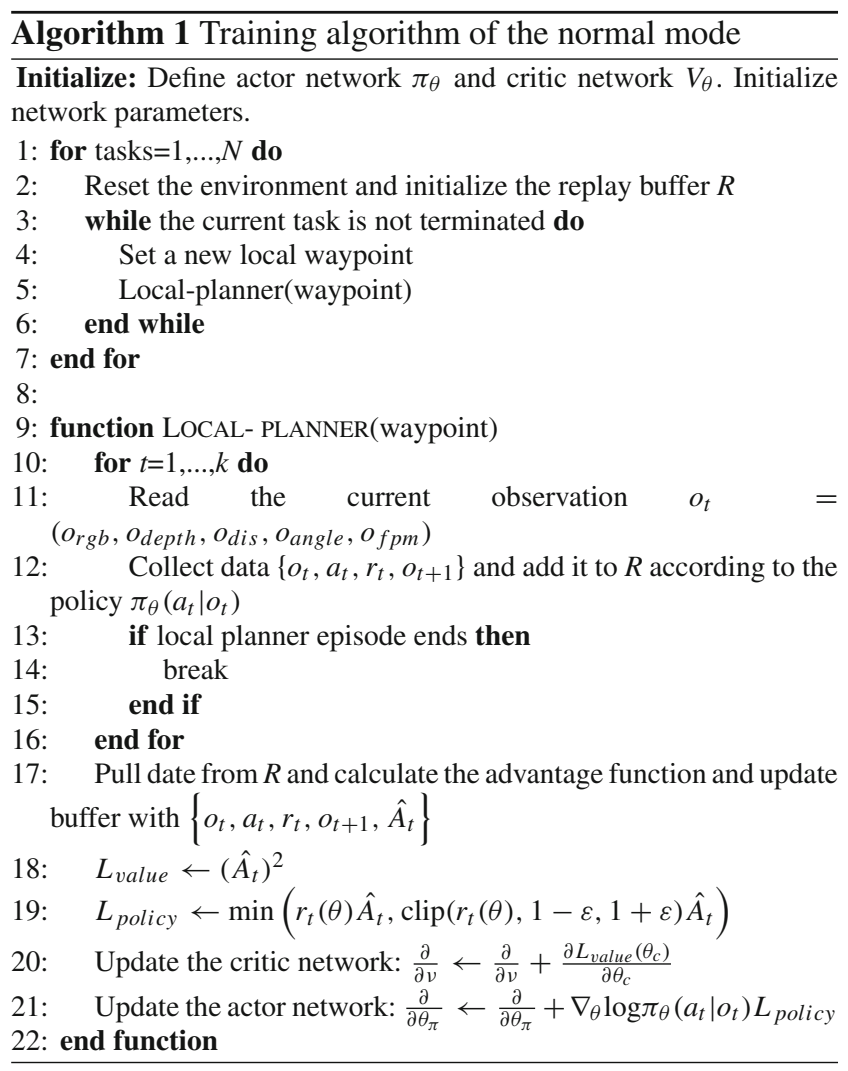

To better avoid possible collisions and tracking failure, we then trained the collision-avoidance mode and the tracking-failure-avoidance mode. During the navigation process, given a local waypoint, we chose the suitable movement mode according to the observation of the robot at each step. When the minimum depth value $d_{\text {min }}^{t}$ in the current depth image was less than the threshold $T h_{\text {depth }}$ for some time steps $D_{\text {depth }}$, we chose to enter the collision-avoidance mode. When the number of feature points $N_{\text {track }}^{t}$ in the current binary feature point image was less than the threshold $T h_{\text {points }}$ for some time steps $D_{\text {points }}$ and the current local waypoint was not the goal, we chose to enter the trackingfailure-avoidance mode. Table 1 gives the concrete parameter values used in our evaluation. We used an Intel Core i910900X processor, with 64 GB RAM and NVIDIA RTX 3090 GPU for model training and testing.

\section{Evaluation metrics}

We used four evaluation metrics: success, success weighted by path length (SPL) [3], average Collision Rate (CR) and average Tracking Failure Rate (TFR). A PointGoal navigation task was considered successful if the robot reached the goal within $0.2 \mathrm{~m}$ without collisions or tracking failure. Given the geodesic distance of the shortest path $l$ and the length of the path $p$ taken by the robot for each task, the popular SPL metric was calculated as follows:

$S P L=\frac{1}{N} \sum_{i=1}^{N} S_{i} \cdot \frac{l_{i}}{\max \left(p_{i}, l_{i}\right)}$,

where $S_{i}$ is the binary indicator of success in the task $i$.

In addition, we can calculate the average Collision Rate (CR) and average Tracking Failure Rate (TFR) by counting the number of collisions and tracking failure.

\section{Simulation setup}

We used the Habitat simulator [28] as our training and evaluation platform, which are 3D reconstructions of real indoor spaces from the Gibson dataset [57]. We used the train split of the dataset from the Habitat repository. We evaluated our approach over five trials across five unseen test environments (see Fig. 7). In the Habitat simulator, we set the camera height to $0.45 \mathrm{~m}$. The spatial resolution of the RGB image and depth image were both set to be $(640,480)$. Considering that many mainstream RGB-D cameras have narrow horizontal field of view $(\mathrm{FoV})$. For example, Kinect v1 has only a horizontal FOV of 57-degree. Therefore, the horizontal FOV was set to be 57-degree, which is very important for practical PointGoal navigation applications.

\section{Results}

\section{Training analysis}

In the training process, we randomly sampled the PointGoal navigation task from the training dataset. In the first 500 tasks, we only trained the normal mode. In the following 1000 tasks, we compared the difference between continuously training the normal mode and freezing the weights of the normal mode to train the other two modes. The comparison results are shown in Fig. 8. For convenience, we define the model trained with and without the collision-avoidance mode and the tracking-failure-avoidance mode as Safe-Nav and Baseline, respectively. 
Table 1 Parameter values

\begin{tabular}{lll}
\hline Parameter description & Parameter & Value \\
\hline Maximum linear velocity & $v_{\text {max }}$ & 0.2 \\
Minimum linear velocity & $v_{\text {min }}$ & 0.1 \\
Angular velocity & $\omega$ & 12 \\
Constant for reaching the local waypoint & $R_{\text {wp }}$ & 20 \\
Distance threshold within the local waypoint is reached & $D_{\text {wp }}$ & 0.2 \\
Weight for the change of relative distance & $\omega_{1}$ & 50 \\
Weight for the change of relative angle & $\omega_{2}$ & 1 \\
Constant for colliding with obstacles & $R_{C O}$ & 20 \\
Constant for tracking failure & $R_{t f}$ & 20 \\
Weight for the change of minimum depth value & $\omega_{3}$ & 50 \\
Weight for the change of average depth value & $\omega_{4}$ & 60 \\
Weight for the change of the number of feature points & $\omega_{5}$ & 0.1 \\
Time steps threshold for continuous back motions & $D_{\text {back }}$ & 3 \\
Constant for continuous back motions & $R_{\text {back }}$ & 5 \\
Time steps of each local planner episode & $k$ & 15 \\
Threshold of the minimum depth value & $T h_{\text {depth }}$ & 0.3 \\
Time steps of entering the collision-avoidance mode & $D_{\text {depth }}$ & 2 \\
Threshold of the number of feature points & $T h_{\text {points }}$ & 280 \\
Time steps of entering the tracking-failure-avoidance mode & $D_{\text {points }}$ & 2 \\
\hline
\end{tabular}
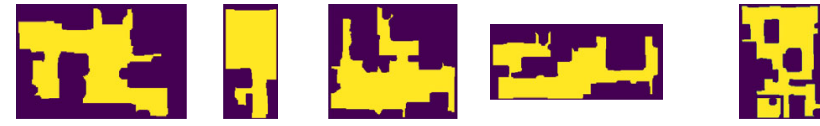

Fig. 7 Simulation test scenes. From left to right: Denmark $\left(41 \mathrm{~m}^{2}\right)$, Edgemere $\left(24 \mathrm{~m}^{2}\right)$, Greigsville $\left(44 \mathrm{~m}^{2}\right)$, Pablo $\left(38 \mathrm{~m}^{2}\right)$ and Ribera (51 $\mathrm{m}^{2}$ )

1. First, we compare the success rate during the training process of Safe-Nav and Baseline. Figure 8a presents the results, where the brown curve represents the training process of Baseline. At the 500th task, the collisionavoidance mode and the tracking-failure-avoidance mode were added to the training process for comparison, shown in the green curve. The success rate of Safe-Nav first decreases and then increases rapidly from the 600th task to the 1500th task. This is because the other two modes were not well trained from the 500th task to the 600th task. After the 900th task, the advantages of adding the collision-avoidance mode and the tracking-failureavoidance mode can be reflected. Safe-Nav has better performance than Baseline from the 900th task to the end of the training.

2. Second, we compare the collision rate during the training process of Safe-Nav and Baseline. Figure 8b shows the comparison results. After adding the collision-avoidance mode, the collision rate of Safe-Nav first increases slightly. As the training continues, the collision rate of Safe-Nav tends to decrease and reaches a minimum when the training ends. However, with the increase of training tasks, the collision rate of Baseline decreases very slowly. There are two possible reasons. On the one hand, compared with the normal mode, there is only one type of spatial input (depth image) in the collision-avoidance mode, which reduces the interference of irrelevant information. On the other hand, the collision-avoidance mode benefits from the specially designed multi-constraint reward function, which further improves the performance of avoiding obstacles.

3. Third, we compare the tracking failure rate during the training process of Safe-Nav and Baseline. Figure 8c shows the comparison results. The tracking failure rate of Safe-Nav first increases from the 500th task to the 700th task, which indicates that the tracking-failure-avoidance mode is unstable in the early stage of the training process. After the 700th task, the tracking failure rate declines significantly and finally achieves almost the same result as Baseline. It is noted that the tracking failure rate is calculated in the whole training process, which reflects the changing trend of the tracking failure rate during the training process, including the early unstable stage of the tracking-failure-avoidance mode. Therefore, the final tracking failure rate of Safe-Nav is actually much lower than Baseline.

The following conclusions can be obtained based on the results described above: 


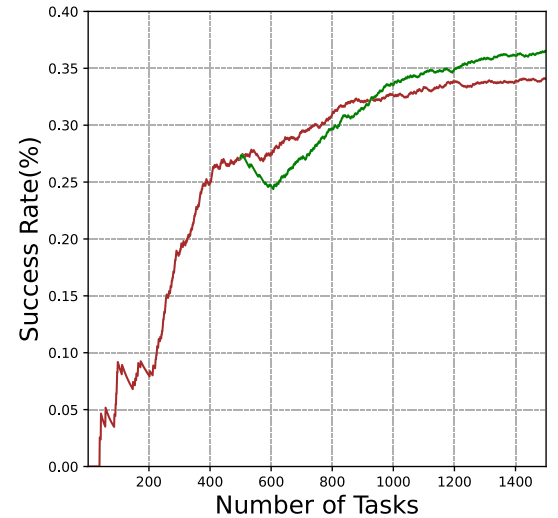

(a)

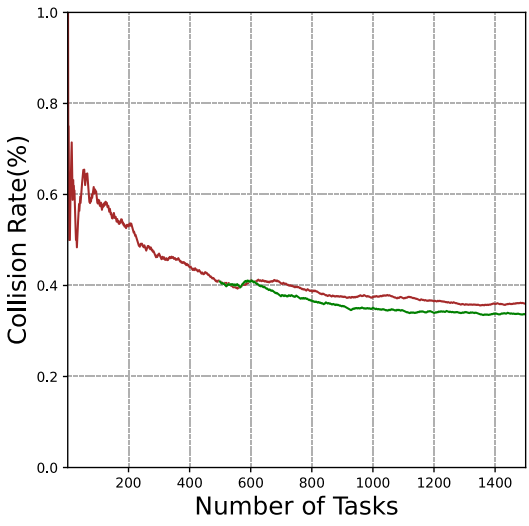

(b)

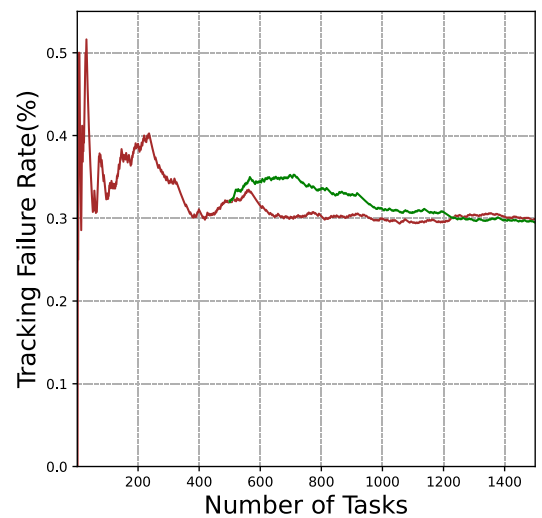

(c)

Fig. 8 The success rate, collision rate and tracking failure rate during training. The green and brown curves are training process of our model trained with and without the collision-avoidance mode and the tracking-failure-avoidance mode

Remark 1 As the number of training tasks increases, the performance of Baseline grows very slowly. This is because a lot of interaction with the environment is needed in the training process of deep reinforcement learning, which increases the cost of training.

Remark 2 In Safe-Nav, benefited from the specialized collision-avoidance mode and the specialized trackingfailure-avoidance mode, the training time is obviously shortened, and the collision rate and the tracking failure rate can be significantly reduced.

\section{Performance evaluation}

\section{Comparison with the existing DRL-based methods}

We compared Safe-Nav with two state-of-the-art DRL-based methods: PPO [28] and DD-PPO [23]. The average success rate, SPL scores and average collision rate are reported in Table 2. Different from the works in [23,28], a PointGoal navigation task using DRL-based methods was considered successful only when the robot reached the goal position without collisions in our research. From Table 2, we can observe that uniformly across the datasets, our method performs best. Our method effectively improves the success rate and SPL scores, while decreasing the collision rate by a large margin. In addition, both the PPO and DD-PPO methods have very high collision rates. During the navigation process, we observed that the robot using the PPO and DD-PPO methods tended to hit the obstacles instead of avoiding them. However, this behavior was not realistic, a real-world robot would bump into obstacles and simply stop upon colliding. In contrast, our method performed much better in most PointGoal navigation tasks.
Table 2 Comparison of DRL-based methods

\begin{tabular}{llll}
\hline Method & Success rate & SPL & Collision rate \\
\hline PPO & 17.92 & 16.88 & 80.83 \\
DD-PPO & 6.70 & 6.60 & 93.30 \\
Safe-Nav & 55.39 & 51.91 & 19.82 \\
\hline
\end{tabular}

$\overline{\text { We report the average success rate, SPL scores and the average collision }}$ rate

\section{Comparison with classic methods}

This section includes an extensive comparison with the wellknown classic local planner DWA. We kept the visual SLAM module and the global planner module unchanged and only changed the local planner module. The representative qualitative trajectories of our Safe-Nav method and DWA on each test scene are illustrated in Figs. 9, 10, 11, 11 and 13. In all the cases, the DWA trajectories are shown in red. The trajectories of our normal mode, collision-avoidance mode and trackingfailure-avoidance mode are shown in blue, green and pink, respectively.

As shown in Fig. 9, the left and right sides of the Edgemere scene contain many featureless areas, such as white walls, increasing the risk of tracking failure. In Fig. 9a, the DWA method selects to move close to a wall and slide along the wall, which leads to a collision eventually. Instead of moving close to obstacles, the proposed method selects to move along the wall at a distance. When the minimum depth value is less than the threshold, the robot will adjust the angle in time and then continue to move forward. In Fig. 9b, there are two major featureless regions in this scene: the white wall in the lower left corner of the starting point and the white wall on the right side of the small room in the lower left corner. The selected path by the DWA method traverses through the 
Fig. 9 Example robot trajectories on the Edgemere test scene

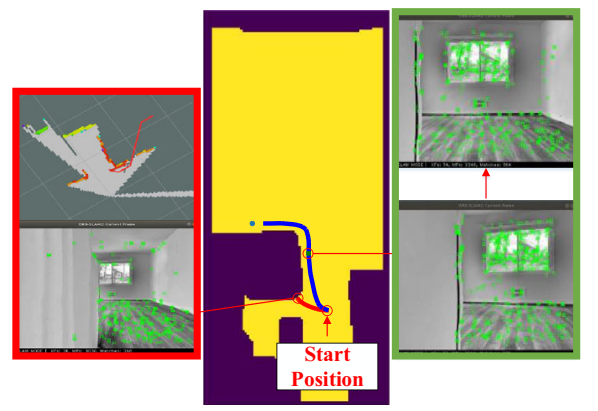

(a)

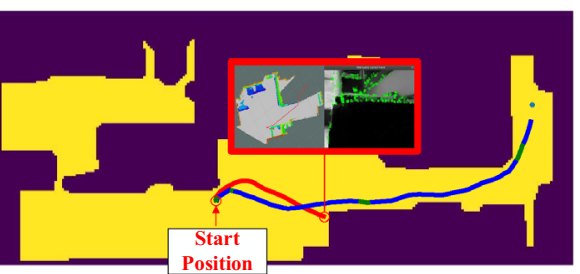

(a)

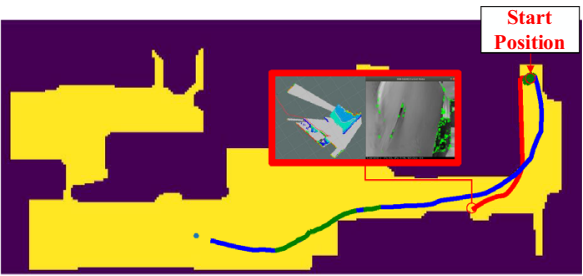

(b)

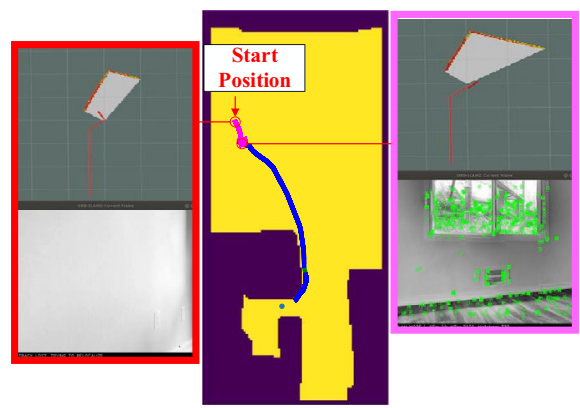

(b)

Fig. 11 Example robot trajectories on the Greigsville

Fig. 10 Example robot trajectories on the Pablo test scene test scene (a)

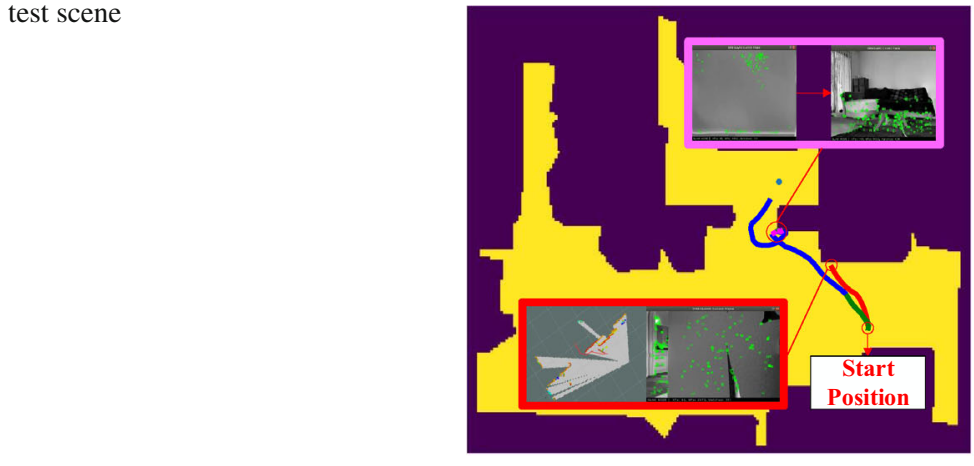

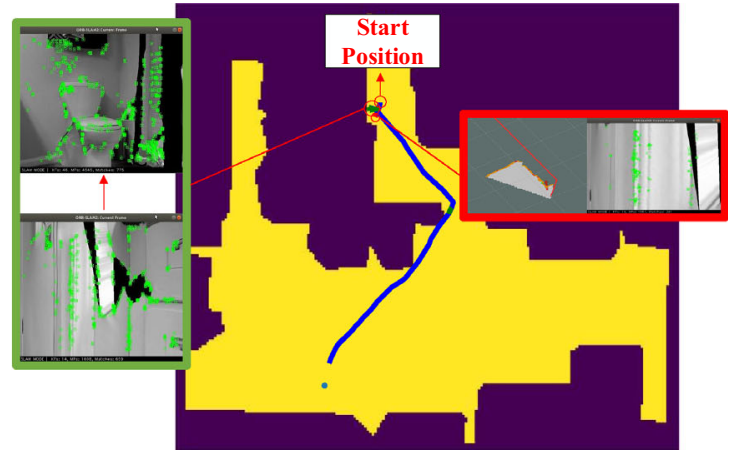

(b) featureless region, which results in tracking failure. In contrast, the robot enters the tracking-failure-avoidance mode around the starting point using the proposed method. Instead of moving close to the local waypoint, the robot chooses to move backward and turn right to get more feature points.

As shown in Fig. 10, the Pablo scene contains two narrow corridors on the right side, which increase the risk of collisions. In Fig. 10a and b, the robot is easy to collide with obstacles at the corner. This may be because DWA only relies on the navigation map and location to plan local paths, and lacks the ability of using rich visual information to perceive surrounding obstacles. Moreover, the estimated navigation map and location often contain errors inevitably, resulting in collisions easily. However, the proposed method can reach the goal position successfully without collisions in both cases, which reflects that it has better ability of avoiding obstacles.

As shown in Fig. 11, the Greigsville scene contains a narrow and featureless corridor on the right side of the center. In Fig. 11a, with the help of the tracking-failure-avoidance mode, the robot successfully passes the featureless corridor by turning one circle to adjust its orientation. However, the robot has already collided with obstacles before it gets close to this corridor using the DWA method. In Fig. 11b, the robot collides in the first few steps using the DWA method. On the contrary, our robot can successfully pass the narrow corridor and finally reaches the goal position.

As shown in Fig. 12, the size of the Ribera scene is large, which includes many featureless areas and narrow corridors, increasing the difficulty of PointGoal navigation tasks. Figure 12a illustrates a long-distance PointGoal navigation task. 
Fig. 12 Example robot trajectories on the Ribera test scene

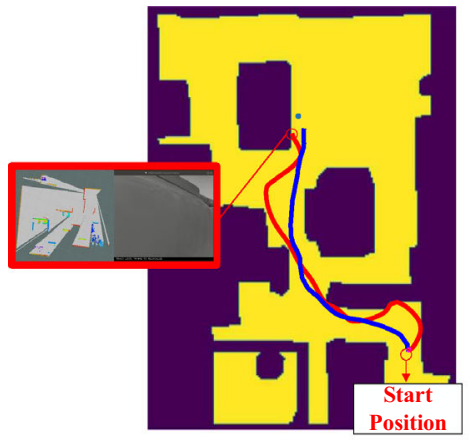

(a)

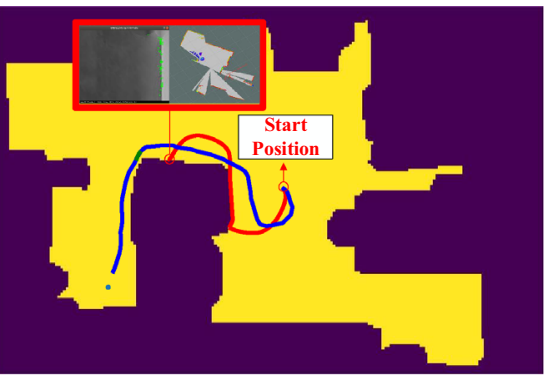

(a)
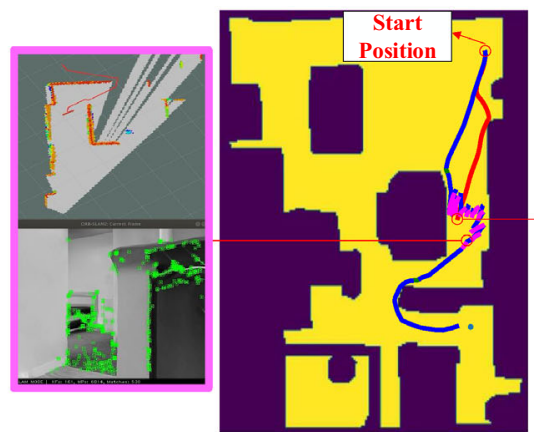

(b)

Fig. 13 Example robot trajectories on the Denmark test scene

Table 3 Comparison with the classic local planner DWA. We report the average success rate (SR), SPL scores, the average collision rate (CR) and the average tracking failure rate (TFR)

\begin{tabular}{|c|c|c|c|c|c|c|c|c|}
\hline \multirow[t]{2}{*}{ Scene } & \multicolumn{2}{|l|}{ SR } & \multicolumn{2}{|l|}{ SPL } & \multicolumn{2}{|l|}{$\mathrm{CR}$} & \multicolumn{2}{|l|}{ TFR } \\
\hline & DWA & Safe-Nav & DWA & Safe-Nav & DWA & Safe-Nav & DWA & Safe-Nav \\
\hline Ed & 19.35 & 45.07 & 17.19 & 41.08 & 19.35 & 15.49 & 61.29 & 38.03 \\
\hline $\mathrm{Pa}$ & 52.17 & 56.25 & 42.09 & 55.18 & 39.13 & 30.21 & 8.70 & 13.54 \\
\hline $\mathrm{Gr}$ & 20.83 & 47.22 & 16.58 & 43.73 & 37.50 & 25.00 & 41.67 & 27.78 \\
\hline $\mathrm{Ri}$ & 8.33 & 45.95 & 5.24 & 41.05 & 41.67 & 16.62 & 50.00 & 37.84 \\
\hline De & 16.67 & 82.35 & 12.96 & 78.51 & 33.33 & 11.76 & 50.00 & 5.88 \\
\hline Total & 23.02 & 55.39 & 18.53 & 51.91 & 33.33 & 19.82 & 43.65 & 24.61 \\
\hline
\end{tabular}

Here, the Edgemere test scene is abbreviated as Ed, the Pablo test scene is abbreviated as Pa, the Greigsville test scene is abbreviated as Gr, the Ribera test scene is abbreviated as Ri and the Denmark test scene is abbreviated as De

The robot finally approaches a featureless sofa, resulting in tracking failure using the DWA method. On the contrary, the proposed method can successfully reach the goal position without collisions and tracking failure. In Fig. 12b, the left side of the start position is a featureless region, which is a white wall. In contrast, many feature-rich regions are distributed along the walls surrounding the environment, especially the walls of the upper and the bottom sides. However, the local waypoint planned by the $\mathrm{A}^{*}$ algorithm is in the featureless region when the robot is at the starting point. Tracking failure occurs when the robot turns left using the DWA method. Instead of naively adjusting the orientation towards the local waypoint, the robot chooses to move backward and turn right to get more feature points.

As shown in Fig. 13, the Denmark test scene contains one major featureless region: the white wall at the end of the longitudinal corridor on the right of the center. In contrast, many feature-rich regions are distributed along the corridor, especially the cabinets of the left and right side. In Fig. 13a, the robot approaches the wall and collides with it using the DWA method. Similar to the previous environments, DWA chooses to naively move towards the local waypoints in Fig. 13b. However, since the robot looks towards featureless regions, it results in tracking failure. On the contrary, the proposed algo- 
Fig. 14 Examples of two common cases in the PointGoal navigation tasks. The robot moves along a planned path shown by black dotted lines, and the images seen at different places are connected with the robot by red dotted lines

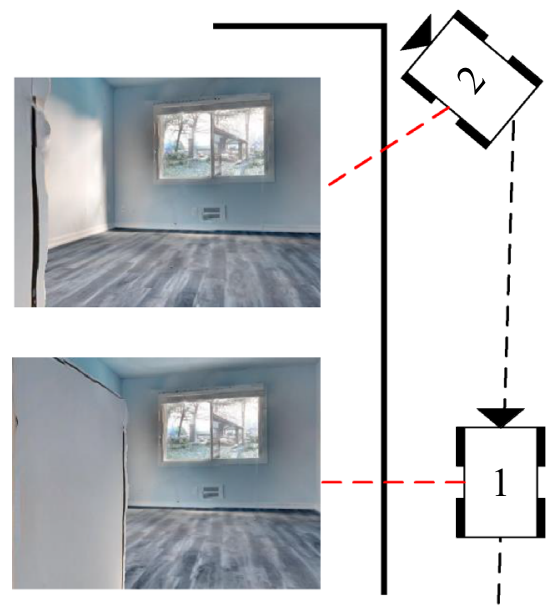

(a)

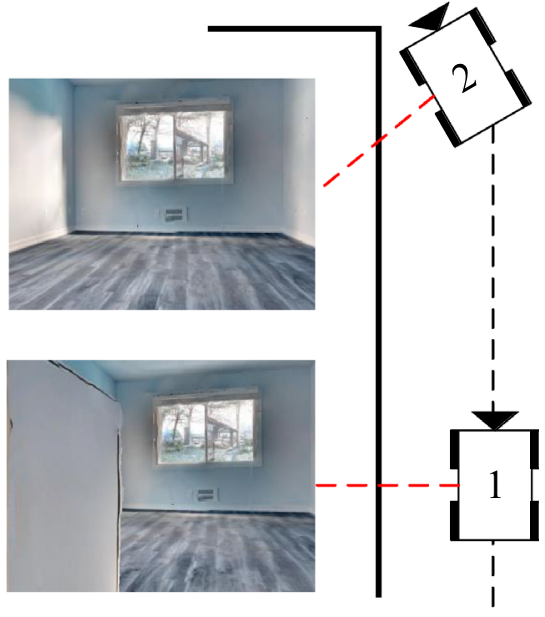

(b)

Table 4 The ablation study of PointGoal navigation results. We report the average success rate (SR), SPL scores, the average collision rate (CR) and the average tracking failure rate (TFR) for Baseline and Safe-Nav

\begin{tabular}{|c|c|c|c|c|c|c|c|c|}
\hline \multirow[t]{2}{*}{ Scene } & \multicolumn{2}{|l|}{ SR } & \multicolumn{2}{|l|}{ SPL } & \multicolumn{2}{|l|}{$\mathrm{CR}$} & \multicolumn{2}{|l|}{ TFR } \\
\hline & Baseline & Safe- Nav & Baseline & Safe- Nav & Baseline & Safe- Nav & Baseline & Safe- Nav \\
\hline $\mathrm{Ed}$ & 37.50 & 45.07 & 37.13 & 41.08 & 18.75 & 15.49 & 43.75 & 38.03 \\
\hline $\mathrm{Pa}$ & 50.70 & 56.25 & 49.71 & 55.18 & 40.85 & 30.21 & 8.45 & 13.54 \\
\hline $\mathrm{Gr}$ & 41.46 & 47.22 & 38.27 & 43.73 & 26.83 & 25.00 & 31.71 & 27.78 \\
\hline $\mathrm{Ri}$ & 26.87 & 45.95 & 25.64 & 41.05 & 28.35 & 16.62 & 44.78 & 37.84 \\
\hline De & 41.67 & 82.35 & 40.28 & 78.51 & 30.00 & 11.76 & 28.33 & 5.88 \\
\hline Total & 39.64 & 55.39 & 38.21 & 51.91 & 28.96 & 19.82 & 31.40 & 24.61 \\
\hline
\end{tabular}

Note: Here, the Edgemere test scene is abbreviated as Ed, the Pablo test scene is abbreviated as Pa, the Greigsville test scene is abbreviated as Gr, the Ribera test scene is abbreviated as Ri and the Denmark test scene is abbreviated as De

rithm generates trajectories traversing feature-rich regions through a sequence of back and forth motions.

Table 3 lists the quantitative evaluations for DWA and the proposed method. In total, we conducted five test runs for each PointGoal navigation task. We evaluate the performance of the local planner by calculating the success rate, the average collision rates with all obstacles, the average tracking failure rates and SPL. The results show that the proposed method outperforms the classic local planner DWA method in terms of all the indicators, and the safety becomes more evident. However, we found that the robot often had trouble passing through narrow featureless corridors, producing dozens of time steps in length. We believe that actively adjusting the RGB-D camera's orientation may help the robot pass through these areas easier. Another limitation was that the robot often easily collided with the corners of obstacles due to the narrow FOV. As Fig. 14a shows, once the robot observes that the depth value is very small, it will immediately enter into the collision-avoidance mode. Then, it will turn right and move forward to avoid obstacles. Collisions can be avoided in many cases after adding the collision-avoidance mode. However, as Fig. 14b shows, the current position of the robot is very close to the obstacles and there are no obstacles in the historical observations along the way due to a narrow FOV. In this situation, the robot is very likely to move forward and collide with obstacles. Regarding this issue, we believe that the proposed method could be further improved with the help of memory modules, which are active areas in the research of computer vision [58,59].

\section{Ablative analysis}

To demonstrate the effectiveness of the collision-avoidance mode and the tracking-failure-avoidance mode, we compared Safe-Nav with an ablated version: Baseline policy which only uses the normal mode without other modes. The summarized results (Table 4) shows that the proposed Safe-Nav performs better than Baseline policy, as it introduces two extra special- 
ized movement modes, which can better deal with avoiding obstacles and featureless areas.

\section{Conclusion}

In this paper, we present and validate a framework SafeNav for planning a path by a mobile robot toward a goal through an unknown environment without collisions and tracking failure. The DRL-based local planner is integrated into the conventional navigation pipeline. We can directly train and deploy DRL-based algorithms as local planners by combining the visual SLAM module and global planner module. First, the method Nav-SLAM is proposed for navigation mapping under complex indoor environments using the RGB-D sensors. Second, the proposed global planner module generates local waypoints. Third, three different movement modes in the local planner module are combined to plan a path free of collisions and tracking failure.

In the future, the end-to-end DRL-based local planner approaches by integrating some long-term memory modules $[58,59]$ will be our focus. Moreover, how to actively adjust the RGB-D camera's orientation to choose the best viewing directions to pass through featureless areas will also be our interest. In addition, DRL is treated as black-box models, lacking interpretability. We believe that more explainable and robust models could be obtained using some explainable AI technologies [60].

Funding This work was supported by the National Key R\&D Program of China (2017YFC0306200), China Postdoctoral Science Foundation (2021M692390), and the Seed Foundation of Tianjin University.

Data Availability Not applicable.

Code Availability Not applicable.

\section{Declarations}

Conflict of interest The authors declare that they have no conflict of interest.

Ethics approval This article does not contain any studies with human participants or animals performed by any of the authors.

Consent to participate Not applicable.

Consent for publication Not applicable.

Open Access This article is licensed under a Creative Commons Attribution 4.0 International License, which permits use, sharing, adaptation, distribution and reproduction in any medium or format, as long as you give appropriate credit to the original author(s) and the source, provide a link to the Creative Commons licence, and indicate if changes were made. The images or other third party material in this article are included in the article's Creative Commons licence, unless indicated otherwise in a credit line to the material. If material is not included in the article's Creative Commons licence and your intended use is not permitted by statutory regulation or exceeds the permitted use, you will need to obtain permission directly from the copyright holder. To view a copy of this licence, visit http://creativecomm ons.org/licenses/by/4.0/.

\section{References}

1. Yasuda YDV, Martins LEG, Cappabianco FAM (2020) Autonomous visual navigation for mobile robots: a systematic literature review. ACM Comput Surv 53:1-34. https://doi.org/ $10.1145 / 3368961$

2. Bonin-Font F, Ortiz A, Oliver G (2008) Visual navigation for mobile robots: a survey. J Intell Robot Syst 53:263-296. https:// doi.org/10.1007/s10846-008-9235-4

3. Anderson P, Chang A, Chaplot DS, Dosovitskiy A, Gupta S, Koltun V, Kosecka J, Malik J, Mottaghi R, Savva M, \& Zamir AR (2018) On evaluation of embodied navigation agents. Preprint at arXiv.org/abs/1807.06757 (2018)

4. Mishkin D, Dosovitskiy A, Koltun V (2019) Benchmarking classic and learned navigation in complex 3D environments. Preprint at arxiv.org/abs/1901.10915

5. Möller R, Furnari A, Battiato S, Härmä A, Farinella GM (2021) A survey on human-aware robot navigation. Rob Auton Syst. https:// doi.org/10.1016/j.robot.2021.103837

6. Blösch M, Weiss S, Scaramuzza D, Siegwart R (2010) Vision based MAV navigation in unknown and unstructured environments. In: 2010 IEEE international conference on robotics and automation (ICRA), pp. 21-28. IEEE, Anchorage, AK, USA . https://doi.org/ 10.1109/ROBOT.2010.5509920

7. Thrun S, Montemerlo M, Dahlkamp H et al (2006) Stanley: the robot that won the darpa grand challenge. J F Robot 33:1-17. https://doi.org/10.1002/rob.20147

8. Hutter M, Gehring C, Jud D, et al (2016) ANYmal—a highly mobile and dynamic quadrupedal robot. In: IEEE/RSJ international conference on intelligent robots and systems (IROS), pp. 38-44. IEEE, Daejeon, South Korea https://doi.org/10.1109/IROS.2016. 7758092

9. Cadena C, Carlone L, Carrillo H et al (2016) Past, present, and future of simultaneous localization and mapping: towards the robust-perception age. IEEE T Robot 32(6):1309-1332. https:// doi.org/10.1109/TRO.2016.2624754

10. Fuentes-Pacheco J, Ruiz-Ascencio J, Rendón-Mancha JM (2015) Visual simultaneous localization and mapping: a survey. Artif Intell Rev 43:55-81. https://doi.org/10.1007/s10462-012-9365-8

11. Dong X, Cheng L, Peng H, Li T (2021) FSD-SLAM: a fast semidirect SLAM algorithm. Complex Intell Syst. https://doi.org/10. 1007/s40747-021-00323-y

12. Hart PE, Nilsson NJ, Raphael B (1968) A formal basis for the heuristic determination of minimum cost paths. Syst Sci Cybern 4:100-107

13. Stentz A (1995) Optimal and efficient path planning for unknown and dynamic environments. Int J Robot Autom 10:89-100

14. Fox D, Burgard W, Thrun S (1997) The dynamic window approach to collision avoidance. IEEE Robot Autom Mag 4:23-33. https:// doi.org/10.1109/100.580977

15. Rosmann C, Feiten W, Wosch T (2013) Efficient trajectory optimization using a sparse model. In: 2013 European conference on mobile robots (ECMR), pp. 138-143. IEEE, Barcelona, Catalonia, Spain . https://doi.org/10.1109/ECMR.2013.6698833

16. Tzafestas SG (2018) Mobile robot control and navigation: a global overview. J Intell Robot Syst 91:35-58. https://doi.org/10.1007/ s10846-018-0805-9 
17. Roesmann C, Feiten W, Woesch T et al (2012) Trajectory modification considering dynamic constraints of autonomous robots. In: the 7th German conference on robotics, $\mathrm{p}$ 1-6

18. Zhong J, Wang T, Cheng L (2021) Collision-free path planning for welding manipulator via hybrid algorithm of deep reinforcement learning and inverse kinematics. Complex Intell Syst. https://doi. org/10.1007/s40747-021-00366-1

19. Chen S, Wang M, Song W et al (2020) Stabilization approaches for reinforcement learning-based end-to-end autonomous driving. IEEE Trans Veh Technol 69:4740-4750. https://doi.org/10.1109/ TVT.2020.2979493

20. Min K, Kim H, Huh K (2019) Deep distributional reinforcement learning based high-level driving policy determination. IEEE Trans Intell Veh 4:416-424. https://doi.org/10.1109/TIV.2019.2919467

21. Han Y, Zhou Q, Duan F (2021) A game strategy model in the digital curling system based on NFSP. Complex Intell Syst. https:// doi.org/10.1007/s40747-021-00345-6

22. Silver D, Huang A, Maddison CJ et al (2016) Mastering the game of go with deep neural networks and tree search. Nature 529:484 489. https://doi.org/10.1038/nature16961

23. From NA, Rames BIF, Wijmans E et al (2020) DD-PPO: learning near-perfect pointgoal navigators from 2.5 billion frames. In: 2020 international conference on learning representations (ICLR)

24. Dobrevski M, Skocaj D (2021) Deep reinforcement learning for map-less goal-driven robot navigation. Int J Adv Robot Syst, 1-13 https://doi.org/10.1177/1729881421992621

25. Bruce J, Sünderhauf N, Mirowski P, Hadsell R, Milford M (2018) Learning deployable navigation policies at kilometer scale from a single traversal. Preprint at arXiv.org/abs/1807.05211

26. Mirowski P, Pascanu R, Viola F et al (2017) Learning navigate in complex environments. In: 2017 international conference on learning representations (ICLR)

27. Ye J, Batra D, Wijmans E, Das A (2020) Auxiliary Tasks Speed up Learning PointGoal Navigation. In: 2020 The Conference on Robot Learning (CoRL)

28. Savva M, Kadian A, Maksymets O et al (2019) Habitat: a platform for embodied AI research. In: 2019 IEEE/CVF international conference on computer vision (ICCV), p 9338-9346. IEEE, Seoul, South Korea https://doi.org/10.1109/ICCV.2019.00943

29. Morad SD, Mecca R, Poudel RPK et al (2020) Sim2real predictivity: does evaluation in simulation predict real-world performance? IEEE Robot Autom Lett 5:6670-6677. https://doi.org/10.1109/ LRA.2020.3013848

30. Sax A, Zhang JO, Emi B et al (2019) Learning to navigate using mid-level visual priors. In: 2019 the conference on robot learning (CoRL)

31. Gordon D, Kadian A, Parikh D et al (2019) SplitNet: Sim2sim and Task2task transfer for embodied visual navigation. In: 2019 IEEE/CVF international conference on computer vision (ICCV), p 1022-1031. IEEE, Seoul, South Korea https://doi.org/10.1109/ ICCV.2019.00111

32. Balakrishnan K, Chakravarty P, Shrivastava S (2021) An A* curriculum approach to reinforcement learning for RGBD indoor robot navigation. Preprint at arxiv.org/abs/2101.01774

33. Chaplot DS, Gandhi D, Gupta S et al (2020) Learning to explore using active neural SLAM. In: 2020 international conference on learning representations (ICLR)

34. Ramakrishnan SK, Al-Halah Z, Grauman K (2020) Occupancy anticipation for efficient exploration and navigation. In: the European conference on computer vision (ECCV), pp. 400418. Springer, Glasgow, UK https://doi.org/10.1007/978-3-03058558-7_24

35. Bigazzi R, Landi F, Cornia M et al (2021) Out of the box: embodied navigation in the real world. In: 2021 19th international conference on computer analysis of images and patterns (CAIP)
36. Morad SD, Mecca R, Poudel RPK et al (2021) Embodied visual navigation with automatic curriculum learning in real environments. IEEE Robot Autom Lett 6:683-690. https://doi.org/10. 1109/LRA.2020.3048662

37. Isele D, Cosgun A (2018) Selective experience replay for lifelong learning. In: the AAAI conference on artificial intelligence, p 33023309

38. Rolnick D, Ahuja A, Schwarz J et al (2019) Experience replay for continual learning. In: advances in neural information processing systems (NIPS). MIT Press, Vancouver

39. Atherton LA, Dupret D, Mellor JR (2015) Memory trace replay: the shaping of memory consolidation by neuromodulation. Trends Neurosci 38:560-570. https://doi.org/10.1016/j.tins.2015.07.004

40. Mur-Artal R, Tardos JD (2017) Orb-slam2: an open-source slam system for monocular, stereo, and rgb-d cameras. IEEE Trans Robot 33:1255-1262. https://doi.org/10.1109/TRO.2017.2705103

41. Fu M, Lu X, Liu T, Yang Y, Li X, Li Y (2015) Real-time slam algorithm based on rgb-d data. Robot 37, 683-692 https://doi.org/ 10.13973/j.cnki.robot.2015.0683

42. Hornung A, Wurm KM, Bennewitz M et al (2013) Octomap: an efficient probabilistic 3d mapping framework based on octrees. Auton Robots 34:189-206. https://doi.org/10.1007/s10514-0129321-0

43. Yao J, Lin C, Xie X et al (2010) Path planning for virtual human motion using improved $\mathrm{A}^{*}$ star algorithm. In: 2010 seventh international conference on information technology: new generations, $\mathrm{p}$ 1154-1158. IEEE, Las Vegas https://doi.org/10.1109/ITNG.2010. 53

44. Schulman J, Wolski F, Dhariwal P et al (2017) Proximal policy optimization algorithms. Preprint at arxiv.org/abs/1707.06347

45. Rublee E, Rabaud V, Konolige K, Bradski G (2011) ORB: an efficient alternative to SIFT or SURF. In: 2011 international conference on computer vision (ICCV), pp. 2564-2571. IEEE, Barcelona https://doi.org/10.1109/ICCV.2011.6126544

46. Taketomi T, Uchiyama H, Ikeda S (2017) Visual slam algorithms: a survey from 2010 to 2016. IPSJ Trans Comput Vision Appl. https:// doi.org/10.1186/s41074-017-0027-2

47. Bustos AP, Chin TJ, Eriksson A, Reid I (2019) Visual SLAM: why bundle adjust? In: 2019 international conference on robotics and automation (ICRA), p 2385-2391. IEEE, Montreal https://doi.org/ 10.1109/ICRA.2019.8793749

48. He K, Zhang X, Ren S, Sun J (2016) Deep residual learning for image recognition. In: 2016 IEEE conference on computer vision and pattern recognition (CVPR), p 770-778. IEEE, Las Vegas https://doi.org/10.1109/CVPR.2016.90

49. Chen T, Gupta S, Gupta A (2019) Learning exploration policies for navigation. In: 2019 international conference on learning representations (ICLR)

50. Furfari FA (2002) Attention is all you need. IEEE Ind Appl Mag 8:8-15. https://doi.org/10.1109/2943.974352

51. Hu J, Shen L, Albanie S et al (2020) Squeeze-and-excitation networks. IEEE T Pattern Anal 42:2011-2023. https://doi.org/10. 1109/TPAMI.2019.2913372

52. Woo S, Park J, Lee JY, Kweon IS (2018) CBAM: convolutional block attention module. In: the European conference on computer vision (ECCV), p 3-19. Springer, Munich https://doi.org/10.1007/ 978-3-030-01234-2_1

53. Luong M, Pham H, Manning CD (2015) Effective approaches to attention-based neural machine translation. In: the 2015 conference on empirical methods in natural language processing (EMNLP), $p$ 1412-1421 https://doi.org/10.18653/v1/D15-1166

54. Wang B, Chen C, Lu CX et al (2020) Atloc: attention guided camera localization. The AAAI conference on artificial intelligence https:// doi.org/10.1609/aaai.v34i06.6608

55. Wang X, Girshick R, Gupta A, He K (2018) Non-local neural networks. In: 2018 IEEE/CVF conference on computer vision and 
pattern recognition (CVPR), p 7794-7803. IEEE, Salt Lake City https://doi.org/10.1109/CVPR.2018.00813

56. Zhang H, Goodfellow I, Metaxas D, Odena A (2019) Self-attention generative adversarial networks. In: international conference on machine learning (ICML)

57. Xia F, Zamir AR, He Z et al (2018) Gibson Env: real-world rerception for embodied agents. In: 2018 IEEE/CVF conference on computer vision and pattern recognition (CVPR), p 9068-9079. IEEE, Salt Lake City https://doi.org/10.1109/CVPR.2018.00945

58. Iscen A, Zhang J, Lazebnik S, Schmid C (2020) Memory-efficient incremental learning through feature adaptation. In: the European conference on computer vision (ECCV), p 699-715. Springer, Glasgow https://doi.org/10.1007/978-3-030-58517-4_41

59. Fini E, Lathuiliére S, Sangineto E et al (2020) Online continual learning under extreme memory constraints. In: the European conference on computer vision (ECCV), pp. 720-735. Springer, Glasgow https://doi.org/10.1007/978-3-030-58604-1_43

60. Holzinger H, Malle B, Saranti A, Pfeifer B (2021) Towards multimodal causability with graph neural networks enabling information fusion for explainable ai. Inform Fusion 71:28-37. https://doi.org/ 10.1016/j.inffus.2021.01.008
Publisher's Note Springer Nature remains neutral with regard to jurisdictional claims in published maps and institutional affiliations. 